\title{
What can reverse causation tell us about demographic differences in the social network and social support determinants of self-rated health in later life?
}

\section{Heather Booth, Pilar Rioseco and Heather Crawford*}

\begin{abstract}
Few studies of the association between social networks (SN), social support (SS), and self-rated health (SRH) address the role of demography in determining that association. Yet demography defines social-structural context, differentiates family from friend networks, and influences network structures. This study examines the SN-SRH association through cross-cutting analyses of four demographically defined groups (Males, Females, Partnered, Unpartnered) and three networks (Family, Friend, Group). By distinguishing between 'healthy' and 'unhealthy' samples, the underlying causal mechanisms are explored. The positive causal effect of SN on SRH is almost entirely confined to the healthy. In this sample, Friend SN is operational among Females and the Partnered, and Group SN is operational among Males. In the unhealthy sample, reverse causation accounts for all but a weak positive effect of Group SN on the SRH of the Partnered, while worse SRH among Females has the causal effect of greater emotional SS through confiding in friends. Among the Unpartnered, only the effect of SRH on confiding in family members is significant. The findings call into question the validity of studies which assume only positive causation, and underline the importance of demographic differentiation of both population and networks for understanding the SN-SRH association.
\end{abstract}

\footnotetext{
${ }^{*}$ Heather Booth (corresponding author), Associate Professor, Australian Demographic and Social Research Institute, Coombs Building \#9, Australian National University, ACT 0200, Australia Email: heather.booth@anu.edu.au

Pilar Rioseco, Research Assistant, Australian Demographic and Social Research Institute, Australian National University, Australia

Heather Crawford, Research Assistant, Australian Demographic and Social Research Institute, Australian National University, Australia
} 


\section{Introduction}

It is widely recognised that social networks play an important role in determining health, well-being, and survival at older ages (Bowling and Grundy 1998; Berkman et al. 2000; Litwin and Shiovitz-Ezra 2011; Fiori and Jager 2012). Most research on this topic focuses on social interactions between the ego and network members and/or the function of the network in providing social support. Different bodies of work refer to different outcomes, use different social network variables, and variously focus on the type of network, on family or friends, on differences by sex, or on narrowly specified populations; leading to a large body of findings that are difficult to assimilate. Clarity is not assisted by a failure in some studies to distinguish between the social network and social support. The extent to which the findings are context-specific often cannot be determined. The fact that most studies rely on cross-sectional data to examine this complex association makes it difficult to establish causation. Distinguishing between causation (social activity begets health) and reverse causation (health begets social activity) in the positive association is further complicated by the likelihood that a negative association will arise from reverse causation (worse health begets more social support and health-related activity). These three causal mechanisms are likely to coexist in any population.

In most studies on this topic, the role of demography is largely ignored. While demographic variables appear in statistical models, they usually serve as controls: their role is to allow an overarching relationship between social networks and health to be revealed. Yet social networks are formed primarily along demographic lines (Kalmijn and Vermunt 2007), and health is differentiated by demographic characteristics (Gjonca et al. 2005; Murphy et al. 2007). It can therefore be expected that demographic variables play an important role in determining the relationship between social networks and health, and that the nature of this relationship might differ among demographic groups.

This exploratory study uses cross-cutting demographic differentiation to further examine and explain the association between social networks and health among older people. It employs a structured analysis of the association by considering, on the one hand, different social contexts through demographically defined groups; and, on the other, different social networks bounded by notions of kith and kin and structured by demographic processes and characteristics. By design, the study seeks to distinguish between the three causal mechanisms in order to further illuminate the demographic differences in the overall association. To our knowledge, the study is unique in distinguishing the causal mechanisms underlying the association between social networks and health.

The data are drawn from a national survey which focuses on the social activity levels and well-being of Australian seniors (Booth et al. 2013). The outcome variable is self-rated health (SRH), a widely used, reliable, and strong predictor of survival which is, however, relatively little understood (Lundberg and 
Manderbacka 1996; Idler and Benyamini 1997; Sargent-Cox et al. 2008; Jylhä 2009). Thus, it is important to seek new insights into the determinants of SRH.

The paper is organised as follows. Section 2 contains background material, including the definitions of and the distinctions between social networks (SN) and social support (SS), a review of previous findings on the SN-SRH association, and a discussion of demography in relation to social networks and the SN-SRH association. The term SN-SRH is used in this paper to refer to the overall association between social networks and self-rated health encompassing social support effects. Section 3 presents the study design, including its aims and scope, a conceptual framework for the study of the SN-SRH association, and a clarification of the causal mechanisms underlying the association. Section 4 describes the data, measures, and statistical methods used. The results are presented in Section 5, and a discussion of the results follows in Section 6.

\section{Background}

\subsection{Social networks and social support}

The notion of the social network (SN) has been credited to Barnes (1954). Social network theory views social communication in terms of network members (nodes) and their relationships (ties), rather than the characteristics of individual agents, and views social behaviour as shaped by the patterns and the quality of relationships (Kadushin 2012). Social networks are defined in terms of network structure and the characteristics of network ties. Network structure includes size (number of members), density (ties per member), boundedness (definition by a particular characteristic, such as family membership), homogeneity (of members with respect to a particular characteristic, such as sex or age), proximity (physical distances among network members), and reachability (ease of access to other network members). The characteristics of ties include the frequency of face-to-face and nonface-to-face contact and of group participation, duration (the length of time two members have known each other), the degree of closeness or intimacy, multiplexity (the number of types of functions a tie serves), and reciprocity (the extent to which functional exchanges are reciprocal) (Scott 2000). Social relationships govern the interpersonal flow of resources, including material goods, feelings, assistance, and information; and thereby shape individual behavioural and emotional responses. The social network perspective thus has relevance for understanding how social relationships relate to personal health and well-being (Smith and Christakis 2008).

Most studies of the SN-SRH association among older people focus on a single network function: social support. (Other functions include social influence, social engagement and attachment, and access to resources and material goods.) Social support (SS) refers to the flow of assistance or resources to the older person from his or her social network which is typically treated as singular and egocentric (see Smith 
and Christakis (2008) for a discussion of network analyses involving more complex, supradyadic effects), and may be instrumental, financial, informational, appraisal, or emotional. Most studies refer to perceived social support (e.g. Ashida and Heaney 2008), but a few use actual support (e.g. Litwin 2006).

The distinction between the social network and social support is rarely maintained in the literature (Smith and Christakis 2008). A multiplicity of terms have been employed, which often span and conflate these two concepts (examples include social engagement, social capital, social connectedness, social interaction, and social environment). Some studies of the SN-SRH association focus entirely on network functions (e.g. Cheng and Chan 2006), while others focus on the network without regard to function (e.g. Ferlander and Mäkinen 2009). Most studies take no particular account of social-structural conditions, which remain implicit.

\subsection{Findings on the SN-SRH association}

This brief review of the main literature on the SN-SRH association among older people first divides the findings into the effect of the social network and the effect of social support, based on the actual measures used. The review then considers studies involving different types of networks and partially differentiated networks, as distinct from multiple exclusive sub-networks. Finally, the social-structural context is considered, as distinct from model covariates. Almost all of the findings in the literature are based on cross-sectional data. Only three of the studies cited employ longitudinal data (as noted below).

Most existing research regarding the role of the social network has indicated that having more social contact is associated with better SRH (e.g. Litwin 2006; Cherry et al. 2013), while having a poor relationship with one's children or seeing one's family or friends less often is associated with worse SRH (Zunzunegui et al. 2004; Garcia et al. 2005). Participation in group activities has also been found to be associated with better SRH (Norstrand et al. 2012), but not in all populations (Pollack and von dem Knesebeck 2004; Nummela et al. 2008). There is little evidence that network size has an effect on SRH.

The findings regarding the role of social support in the SN-SRH association are mixed. Having greater actual support from adult children and the residential proximity of children have been found to be associated with worse SRH (Litwin 2006). Other studies have shown that having greater perceived social support is associated with better SRH (Dupertuis et al. 2001; Melchior et al. 2003 based on longitudinal data; White et al. 2009; Burke et al. 2012; Gilmour 2012). Analyses by sex of perceived non-instrumental support found no association with SRH in a Chinese sample (Cheng and Chan 2006), but a positive association among women in England (Grundy and Sloggett 2003) and among Japanese men (Okamoto and Tanaka 2004). It is possible that these differences have a cultural basis.

Some studies approach the analysis of the SN-SRH association through the concept of network type, whereby ego's overall network is first classified into 
one of several data-derived types which then form the basis of association with SRH. Several robust types have been identified across studies: 'friend-focused' networks are associated with better SRH, and 'restricted' (referring to low levels of receiving and giving support) networks with worse SRH (Fiori and Jager 2012 based on longitudinal data). Other studies partially differentiate between sub-networks (e.g. network structures are differentiated but network functions are not). Litwin (2006) found that contact with friends had a positive effect on SRH, whereas contact with neighbours had no effect, concluding that 'the autonomy and control that are expressed in the selection of friends seem to contribute to better selfrated health' (p. 351). The voluntariness of friendship has been similarly cited in explaining why social activities with friends have a more positive effect on subjective well-being than social activities with family members (Huxhold et al. 2013 based on longitudinal data). Few studies have considered ego's network as a series of exclusive sub-networks, despite the early and ongoing recognition of the need to distinguish between family and friends (Antonucci and Akiyama 1987a; Lennartsson 1999). Zunzunegui et al. (2004) considered friends, extended family, and children, and found that while a stronger network was associated with better SRH for all three sub-networks, the association was attenuated in the presence of disability only for the network of friends.

While this variation in findings by population points to the importance of the social-structural context in the SN-SRH association, it has so far received little attention (Berkman et al. 2000). The existing contextual comparisons include studies of different countries (Pollack and von dem Knesebeck 2004) and of different ethnic or geographic populations within a country (Zunzunegui et al. 2004; Litwin 2006; Nummela et al. 2008). Kavanagh et al. (2006) used the social characteristics of the local environment as a social-structural context. While several studies have compared the sexes, often finding differences between men and women in the SN-SRH association (e.g. Ferlander and Mäkinen 2009), few have treated gender as explicitly contextual (Kavanagh et al. 2006).

\subsection{Demography and the SN-SRH association}

Demography features in three distinct but interrelated ways in the discussion of social networks. First, demography delineates family (kin) and friend (kith) subnetworks, and their exclusivity allows for contrasts to be drawn in the SN-SRH association. Family and friend networks exhibit different degrees of closeness to ego, and play different roles in the provision of social support (Antonucci and Akiyama 1987a; Seeman and Berkman 1988). Second, demography plays a key role in defining network structures, including those of the family, which is traditionally the most influential social network in human society. Demographic processes are the basis of the family, shaping family network size and other structural characteristics (Wolf 1994). The demographic life course events, such as entering and leaving a partnership and family formation, of both ego and his or her family members 
are instrumental in shaping ego's kinship networks in later life (Antonucci and Akiyama 1987a; Wenger et al. 2000). Demographic and life course factors also feature strongly as dimensions along which friend networks are formed. Studies of the non-kin social networks of adult egos show considerable homogeneity of network members with respect to sex, partnership status, and age; and of their interactions (McPherson et al. 2001; Kalmijn and Vermunt 2007). Group networks may also be homogeneous with respect to demographic variables. For example, certain interests may be gendered, and certain day-time activities may be accessed primarily by retirees (most of whom are older).

Third, demography is an important source of variation within the population. Different demographic groups tend to have networks with different characteristics, resulting from different stages or trajectories in the life course or from different social and cultural norms and values, including different degrees of volition in determining who counts as kin (Finch and Mason 1993). In line with demographic homophily (the tendency of individuals to associate with others of the same kind), differences are found in the network characteristics of men and women (Antonucci and Akiyama 1987b; Ajrouch et al. 2005; McLaughlin et al. 2010), and of groups defined by marital status and by age (Kalmijn and Vermunt 2005). Clearly, social sub-network characteristics and demographic groups are inter-related through demographic boundedness and homophily. These inter-relationships also extend to the characteristics of ties and to social support.

Sex and partnership status (and their interaction) are important determinants of social networks and social support. Sex (or gender) has been extensively cited as a distinguishing factor: women's networks are larger, more supportive, and more multifaceted; and involve more friends, more confidants, and greater intimacy and disclosure; while men's networks emphasize sociability, instrumentality, and activity, and involve few intimate relationships (Antonucci and Akiyama 1987b; Shye et al. 1995). Antonucci et al. (1998) found that among older married couples with children, women reported having more close relationships than men. Partnership status has received less attention (Adams et al. 2011), though it is influential in shaping social networks, especially through childbearing, in sex-specific ways (Wenger et al. 2000).

Age also influences social networks through the stage in the life course (Ajrouch et al. 2005). Age (or birth cohort) may also reflect historical changes in social behaviour and attitudes. Importantly, older people's social networks generally become smaller over time (Bowling and Grundy 1998). This accords with socioemotional selectivity theory (Carstensen 1995): a greater sense of limited remaining life expectancy results in greater selectivity in favour of emotionally rewarding relationships. The voluntariness of friendship would allow for greater selectivity in friend networks than in family networks. Though the family network remains the main source of support (Antonucci and Akiyama 1987a), the reduced friend network is also important in terms of social activity and emotional support (Huxhold et al. 2013). 
Given its role in shaping social networks, and hence social support, demography can be expected to have a significant effect on the relationship between social networks and outcomes such as health. It is therefore important to give full recognition to demographic factors in examining the SN-SRH association. A distinction must be clearly drawn here between separately considering complementary populations (such as males and females) and relegating the distinguishing variable (i.e. sex of ego) to the status of covariate. Most (if not all) studies include covariates in their models to take account of confounding influences. However, the inclusion of demographic variables as covariates does not give sufficient recognition to, and thus cannot adequately illuminate the context. Only by comparing different populations or complementary groups within a population can the influence of different demographic or social-structural contexts be understood. Treatment as a covariate (sex of ego) removes the effect, whereas treatment as a context definition (male vs. female) focuses on the effect. To fully take account of demographic influences, it is necessary to focus on demographic groups. The importance of independent analyses for demographic groups has been previously recognised (Antonucci and Akiyama 1987a; Berkman et al. 2000; Fiori and Jager 2012).

\section{Study design}

\subsection{Aims and scope}

The aim of this exploratory study is the illumination of the SN-SRH association in a national sample of Australian adults aged 50-89. This is done through demographically cross-cutting comparative analyses of the SN-SRH association, while also accounting as far as possible for three underlying causal mechanisms. Thus, three different dimensions are addressed. Specifically, the analysis explores social-structural differences due to sex and partnership status in the underlying causal mechanisms of the association between SRH and three exclusive social networks (family, friend, and group) providing two social support functions (instrumental and emotional).

\subsection{Conceptual framework}

In a landmark paper, (Berkman et al. 2000) proposed a comprehensive conceptual framework detailing how social networks influence health. In this framework, social networks are situated within the social-structural conditions of the macroenvironment, which include culture, socio-economic conditions, politics, and social change. These factors condition the nature of social networks, which are defined in terms of network structure and the characteristics of ties. Social networks provide opportunities for five network functions: social support, social influence, social 
Figure 1:

Conceptual framework linking social networks and health

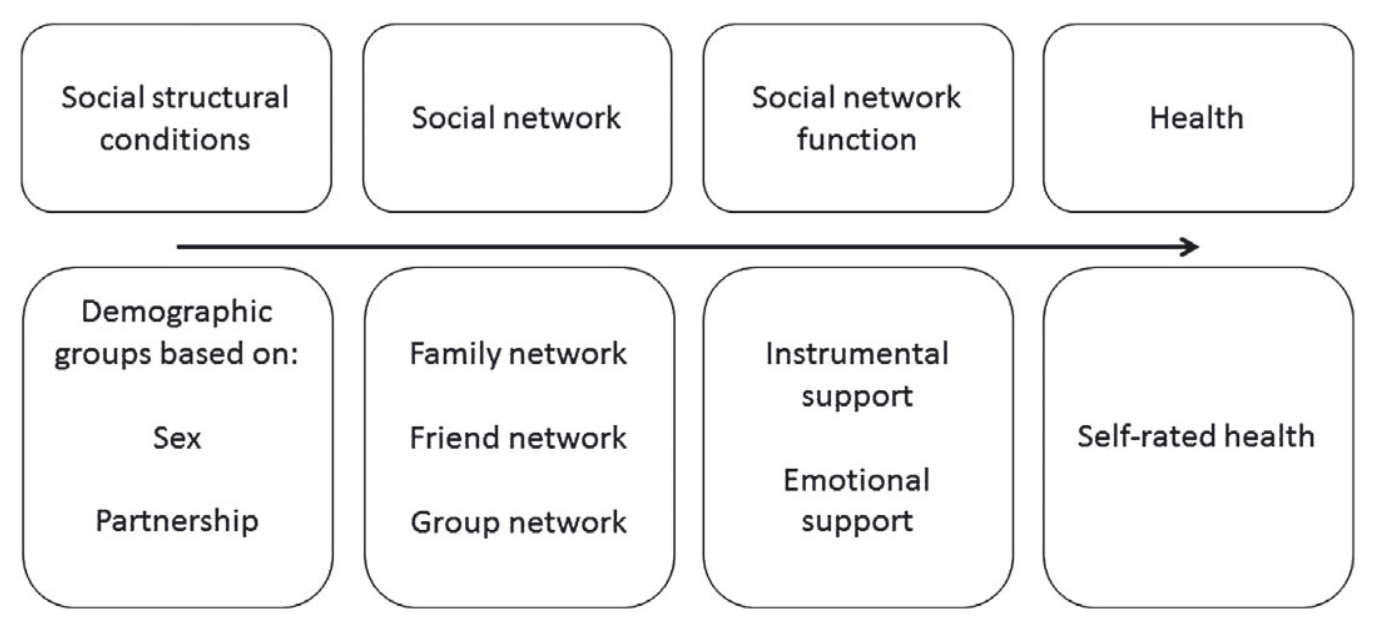

Source: Adapted from Berkman et al. (2000).

engagement, person-to-person (close) contact, and access to resources. These in turn operate to influence health through three pathways: health behaviour, psychology, and physiology. Further details are listed under each aspect of each component.

In this study, we (initially) adopt a conceptual framework (Figure 1) based on the first three components of the Berkman-Glass-Brissette-Seeman (BGBS) framework (pathways are not considered) and the availability of variables. The BGBS framework naturally accommodates demographic factors in both the first and the second components: i.e. the social-structural conditions and the social network. Demographically defined groups can be viewed as representing social-structural conditions within the wider conditions of the relevant society. We individually model four demographic groups in Australian society: Males, Females, Partnered, Unpartnered, and Total.

The social network is also largely demographically defined through network boundedness and demographic homophily. Three exclusive sub-networks are considered: Family, Friend, and Group. These sub-networks are modelled simultaneously, enabling us to determine the net effects of each sub-network and to compare and contrast them. The social networks are egocentric and described in terms of network structure and ties. For the Family and Friend networks, we combine a structural dimension indicating size with tie characteristics indicating the frequency of face-to-face contact; whereas for the Group networks only the frequency of face-to-face contact is used. Two social network functions feature in the framework: Instrumental and Emotional support. These refer to support provided specifically by the Family and Friend networks; thus, network functions are also bounded.

By incorporating demographic variables in the first and second components, cross-cutting demographic differentiation is incorporated in the analytical design: 
the multi-network model is estimated for each of four pairwise-complementary demographic groups. Making comparisons between and within these models can be expected to enhance our understanding of the SN-SRH association.

\subsection{Causal mechanisms}

A defining feature of the BGBS framework is the direction of flow. The components are labelled as 'upstream' and 'downstream' in relation to the social network/function (Berkman et al. 2000). The association is depicted as positive and causal: i.e. a stronger SN begets better health. It is, however, possible that the positive association is due to reverse causation: i.e. better health begets a stronger SN. Moreover, there may also be a negative association, which is logically confined to reverse causation: i.e. being in worse health begets a stronger SN because the individual has a greater need for and is the recipient of SS (whereas being in better health begets a weaker SN through the lack of need for support). (Causation is considered illogical: stronger (weaker) SS is unlikely to lead to worse (better) health.) Disentangling the precise effects of these three causal mechanisms is difficult, and can only be done through a longitudinal study design.

In this study, two strategies are used to isolate, to the extent possible, one or more causal mechanisms of the SN-SRH association. The intent is to allow more meaningful interpretations of this association, and facilitate comparisons across demographic groups and sub-networks. The first strategy follows the structure of the analytical design: clear distinctions are maintained between the social network and social support, between instrumental support and emotional support, and between the three sub-networks. Symmetry of cross-cutting differentiation is also maintained, both between the Family and Friend networks and among demographic groups. This structured analytical design, shown in Tables 3-5, is intended to assist interpretation.

The second strategy is the elimination of one or more mechanisms through subsample selection. We take advantage of the logical selectivity of causal mechanism offered by the variable 'frequency (degree) of social activity restriction due to own (poor) health or disability' (hereafter, 'SN restriction'; see Section 4.2.4). The sample was divided into an 'Unrestricted SN' sample and a 'Restricted SN' sample according to whether the respondent's social activity was never or ever restricted.

By definition, the Unrestricted SN sample does not experience positive reverse causation (health begets $\mathrm{SN}$ ), as the frequency of the $\mathrm{SN}$ restriction is 'never' (see Discussion). Further, because these respondents are not in need of disability-related visits/activities or instrumental support, the strength of their social networks or Instrumental SS is not attributable to negative reverse causation. However, having stronger Emotional SS may stem from having worse health of a form that does not restrict social activity. The logical causal mechanisms for this sample are therefore positive causation, as embodied in the BGBS framework, and negative reverse causation related to Emotional SS. The applicability of causal mechanisms in the SN-SRH association is shown in Table 1. 
Table 1:

Causal mechanisms underlying the SN-SRH association in the Unrestricted SN and Restricted SN samples

\begin{tabular}{|c|c|c|c|}
\hline Causal mechanism & Description & Unrestricted SN sample & Restricted SN sample \\
\hline $\begin{array}{l}\text { Positive causation } \\
\text { SN/SS } \rightarrow \text { SRH }\end{array}$ & $\begin{array}{c}\text { Stronger SN/SS begets } \\
\text { better SRH } \\
\text { Weaker SN/SS begets } \\
\text { worse SRH }\end{array}$ & $\checkmark$ & $\checkmark$ \\
\hline $\begin{array}{c}\text { Positive } \\
\text { reverse causation } \\
\mathrm{SRH} \rightarrow \mathrm{SN} / \mathrm{SS}\end{array}$ & $\begin{array}{c}\text { Better SRH begets } \\
\text { (facilitates) stronger } \\
\text { SN/SS } \\
\text { Poorer SRH begets } \\
\text { weaker (restricts) } \\
\text { SN/SS }\end{array}$ & $\mathrm{X}$ & $\checkmark$ \\
\hline $\begin{array}{l}\text { Negative } \\
\text { reverse causation } \\
\mathrm{SRH} \rightarrow \mathrm{SN} / \mathrm{SS}\end{array}$ & $\begin{array}{l}\text { Poorer SRH begets } \\
\text { stronger SN and } \\
\text { stronger SS } \\
\text { (Instrumental or } \\
\text { Emotional) }\end{array}$ & $\begin{array}{c}\text { SN : X } \\
\text { Instrumental SS: X } \\
\text { Emotional SS: } \checkmark\end{array}$ & $\checkmark$ \\
\hline
\end{tabular}

Note: SN = social network; SS = social support; SRH = self-rated health. The Unrestricted SN and Restricted SN samples include respondents whose social activities are 'never' and 'ever' restricted by their health or disability, respectively.

In contrast, all three causal mechanisms are likely to operate in the Restricted SN sample (see Table 1) Among those with some degree of social restriction due to their own health or disability, a stronger SN is likely to beget better health (positive causation), while worse health is likely to beget both a weaker SN (positive reverse causation) and a stronger SN, through disability-related visits/activities and stronger Instrumental and Emotional SS (negative reverse causation). However, the variable used to divide the total sample can also be used to take account of the degree of social activity restriction due to health or disability in the Restricted SN sample: in other words, reverse causation can be (largely) taken into account. As in the Unrestricted SN sample, the only reverse causation that then logically remains applicable is negative reverse causation due to stronger Emotional SS stemming from worse health (after the level of health/disability has been accounted for by the variable $\mathrm{SN}$ restriction).

\subsection{Health, $\mathrm{SRH}$, and $\mathrm{SN}$ restriction}

It is important to make distinctions between health, SRH and the degree of SN restriction due to health. The three causal mechanisms underlying the SN-SRH 
association refer to health in the general sense of the term. SRH is the indicator of health used as the dependent variable in the analyses. The meaning of SRH is the subject of ongoing research but it is clear that it is not synonymous with medical notions of health (Duncan and Frankenberg 2000; Benyamini et al. 2003; Manderbacka 2013). Rather, SRH is an assessment of health made on the basis of multifarious conditions in the context of possible age, social and temporal comparisons (Sargent-Cox et al. 2008).

In the context of the present study, a positive association between social networks and health, whether due to causation or reverse causation, is more readily appreciated in terms of SRH. In comparison, a negative association, which would arise from reverse causation (worse health begets stronger SS and a stronger SN), is more easily envisaged in terms of physical health and instrumental support. To some extent, causation and reverse causation are likely to be addressing different concepts of health.

The frequency of social activity restriction due to ego's health or disability ( $\mathrm{SN}$ restriction) is the second indicator of health available to the study. SN restriction differs from SRH in two important respects. First, SN restriction is highly likely to be viewed by respondents in terms of physical health, especially given the reference to disability. It is thus much less comprehensive than SRH, omitting health conditions that do not restrict social activity, and ignoring the broader scope of SRH. Second, SN restriction refers to the frequency of restriction due to health/disability rather than to the state of health per se. In the total sample, the correlation between SN restriction and the five-category SRH is 0.53 , indicating only moderate correspondence.

The distinction between SN restriction and SRH is important for understanding the SN-SRH association (See Section 6.5). Greater social restriction due to ego's health/disability implies that ego's social network is increasingly reliant on others. Rather than acting as his or her own social agent, the disabled ego is often a more passive social 'recipient'. This interpretation is consistent with Litwin's observation that network choice and independence are important for (SRH 2006).

\section{Data and methods}

\subsection{Data}

The data are from the 2010/11 national survey of the Social Networks and Ageing Project (SNAP), which was designed to investigate the role of social networks in successful ageing (Booth et al. 2013). Participants were drawn from the membership of National Seniors Australia (NSA), a broad-based Australia-wide older persons' interest group (http://nationalseniors.com.au/). More than half of the group's members reported joining NSA 'to be informed about over 50s issues' (personal communication, NSA). The sampling frame comprised two exclusive membership 
sub-lists which the NSA uses to maintain contact with its members (by email or by post). Each sub-list was stratified by sex and ten-year age group (50 to 89). To avoid sampling both partners of couples, an age-sex-matched substitute was selected. To overcome potential gender bias within couples (arising from the NSA membership numbering system), the initial invitee was asked to assign the invitation to the partner (of the couple) with the more recent birthday (Salmon and Nichols 1983). The postal questionnaire was sent to 2,500 members, and a further 10,000 members were sent an email invitation to complete the questionnaire online. All of the invitees had the option of completing the questionnaire using the alternative method. Overall, the response rate was $17.0 \%$, with approximate (due to uncertain denominators) response rates of $39.4 \%$ for the postal method and $11.4 \%$ for the online method. The final sample of 2,122 Australians comprised $46 \%$ who completed the postal questionnaire and 54\% who responded online. An effect-size metric (Lindenberger et al. 2002) was used to compare the postal sample with the online sample on study variables. Generally, small effect sizes $(<0.20 \mathrm{SD}$ units $)$ indicated no substantial differences between respondents according to mode of completion. Educational differences were somewhat greater $(0.30 \mathrm{SD}$ units). Item response rates were high: dependent variable $>99 \%$, covariates $>97 \%$, and independent variables 78\%-96\%

Compared with the 2011 Australian population (Australian Bureau of Statistics, 2011), the sample under-represents the age groups 50-54 (because NSA membership eligibility starts at age 50) and 80-89 (due to membership attrition and possible age-related non-response), and over-represents the age group 65-69. The sample is, on average, more educated than the general population. Unweighted data are used, since age and education are taken into account in the analysis. The age, sex, and partnership status distribution of the total analytical sample $(n=1522)$ is shown in Figure 2. The group sample sizes are shown in Table 2.

The division of the sample into Unrestricted SN and Restricted SN samples yielded sample sizes of 800 and 722, respectively. The group sample sizes within these samples are shown in Tables 4 and 5, respectively.

\subsection{Measures}

The dependent variable, SRH, is a multidimensional evaluation of the ego's current state of health. At its core is objective health and functioning, but more subjective and contextual factors are also involved (Jylhä 2009). In this study, we employ 'global' SRH, based on the question 'In general, would you say your health is ...?' 1 'Excellent', 2 'Very good', 3 'Good', 4 'Fair', 5 'Poor'. For this study, responses were coded 0 'Excellent/very good/good' and 1 'Fair/poor' for the total sample and the Restricted SN sample, and 0 'Excellent/very good' and 1 'Good/fair/poor' for the Unrestricted SN sample. This distinction was necessary because in the case of the Unrestricted SN sample, the number of respondents with fair/poor SRH in the analytical sample was too small $(n=17)$ for reliable estimation; in fact, no respondents in the Unrestricted SN sample reported having poor health. As the two 
Figure 2:

Age, sex and partnership status distribution of the analytical sample
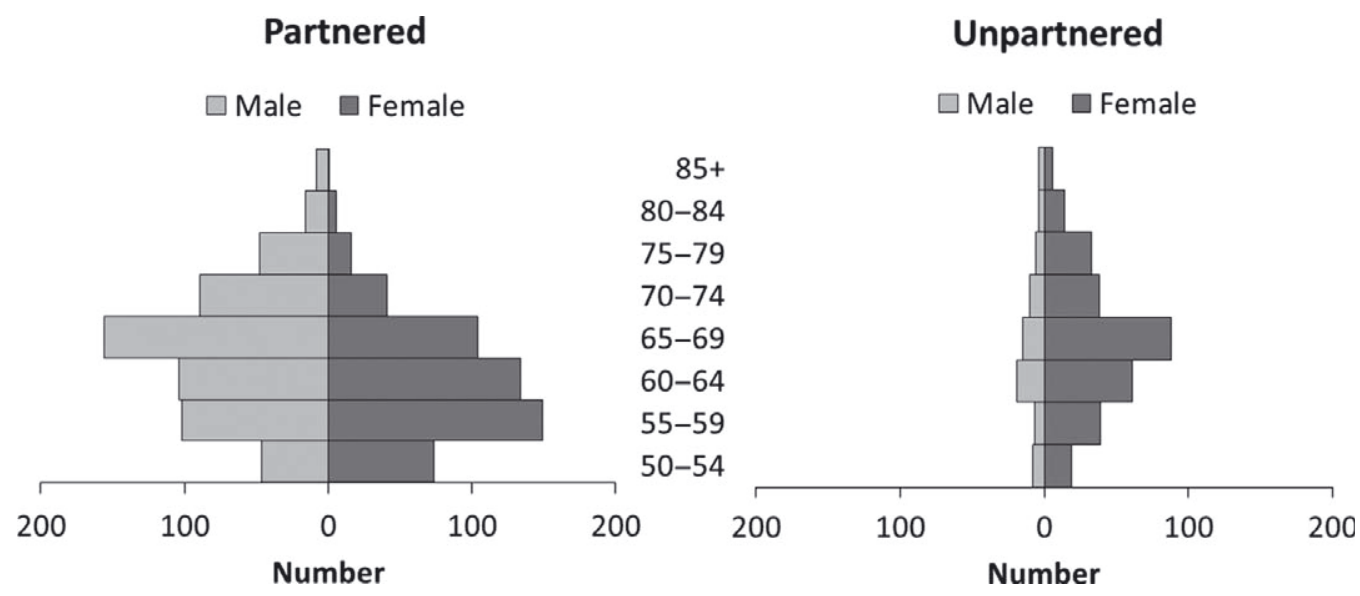

samples are not directly compared, this was considered the optimum solution. The alternative was to model good/fair/poor SRH throughout; this was rejected on the grounds that fair/poor SRH is the main focus in the literature.

The independent variables include social network and social support characteristics referring to the previous four weeks, taken as representing the respondent's usual experience of his or her 'effective' network. Social support is thus actual, rather than perceived. In all cases, the term 'persons' refers to adults aged 18 or older, and the term 'family' refers to adult family members not living in the same household as the respondent. Personal socio-demographic characteristics are included as covariates.

\subsubsection{Social network}

Family network and friend network. Network strength is a continuous variable constructed as the average of three variables: network size, occasions and duration. Network size refers to the number of persons (family or friends) with whom the respondent had been in face-to-face contact, measured on a scale from 1 to 5; coded 1 ' 1 ', 2 ' 2 or 3', 3 ' 4 to 6', 4 '7 to 10', and 5' '11 or more'. Occasions on which the respondent spent time with these family/friends was coded 1 'Once', 2 ' 2 or 3', 3 ' 4 to 6', 4 ' 7 to 10 ', and 5 ' 11 or more'. Duration or time spent on these occasions was coded 1 'Less than an hour', 2 ' 1 hour to less than 5 hours', 3 ' 5 hours to less than 10 hours', 4 ' 10 hours to less than 20 hours', and 5 ' 20 hours or more'. Respondents who had not been in face-to-face contact with any family/friend were coded 0 on all three variables.

Group network. Time spent in group activities ranges from 1 'Less than one hour', to 2 ' 1 to 5 hours', 3 '5 to 10 hours', 4 ' 10 to 20 hours', and 5 ' 21 hours or more'. 
Examples of group activities are committee meetings, gym class, and choir practice. This variable is treated as continuous.

\subsubsection{Social support}

Actual social support characteristics apply to the family/friend networks. Instrumental support was measured by the question: 'How many family/friends assisted you to do something practical?' Emotional support was measured by the question: 'How many family/friends did you confide in?' Both variables are coded 1 '0', '2', 3 '2', 4 ' 3 ', 5 '4', 6 '5-6', and 7 '7+', and are treated as continuous.

\subsubsection{Covariates}

Several covariates are included in the models. Sex and partnership status are taken into account as being relevant within each demographic group. Sex is coded 1 'Male', 2 'Female'. Partnership status is coded 1 'Partnered' if the person was living with a spouse or a de facto partner (or was in a relationship but not living together); and otherwise 0 'Unpartnered'. Age is measured in single years. Other covariates include education and comfortable with standard of living (Kennedy et al. 1998; Mirowsky and Ross 2008). Highest educational qualification is coded 1 'No secondary school qualification', 2 'School certificate/intermediate certificate', 3 'Higher school certificate/trade apprenticeship', 4 'Certificate/diploma', and 5 'University degree or higher'. The respondent's agreement with the statement 'I am comfortable with my standard of living' (on a scale from 1 'Strongly disagree' to 5 'Strongly agree') is used as a proxy measure for socio-economic status. Education and comfortable with living standard are treated as continuous.

\subsubsection{Frequency of social activity restriction due to health or disability}

The survey questionnaire included a question asking: 'How often are your social activities with adult family members (who do not live with you) restricted by your health or disability?' The same question was asked regarding friends. The questions do not refer to a specific time period. Responses range from 1 'Never' to 5 'Always'. Frequency of social activity restriction due to health or disability (SN restriction) is the sum of these two variables, with a score ranging from 2 'Social activities never restricted' to 10 'Social activities always restricted'. As noted in Section 3.3, SN restriction is used to divide the sample into an Unrestricted SN sub-sample (a score of 2, or never restricted) and a Restricted SN sub-sample (a score of 3 to 10, or ever restricted). This variable is also used in modelling SRH in the Restricted SN sub-sample, where it is included to take account of reverse causation. SN restriction provides a direct measure of positive reverse causation and serves as an indicator of 
negative reverse causation, and is thus the key variable in 'isolating' the underlying causal mechanisms in the SN-SRH association.

\subsection{Method}

Logistic regression is used to identify the predictors of worse SRH, using STATA 12. The models are estimated separately for each demographic group, across which the variables are identical, facilitating the comparison of groups. Two models are estimated (as appropriate). Model 1 includes the covariates and independent SN and SS variables. This model is estimated for the total sample and for the Unrestricted SN and Restricted SN samples. Model 2 also includes SN restriction, and is estimated for the Restricted SN sample.

As the purpose of the analysis is to compare the nature of associations among demographic groups and sub-networks, the reporting of regression results is limited to the statistical significance of variables. Thus, in presenting these models in Tables 3-5, '+' indicates that weaker SN or SS is associated with worse SRH (or, equally, stronger SN or SS is associated with better SRH), equivalent to a positive SN-SRH association. Similarly, '-' indicates that stronger SN or SS is associated with worse SRH; in other words, a negative SN-SRH association applies, logically, as negative reverse causation.

\section{Results}

The distributions and the means of the study variables by demographic group are shown in Table 2. In the analytical sample, $14 \%$ rated their health as fair/poor. Associations with sex and with partnership status were found to be non-significant. Logistic regression models (Tables 3-5) show that the SN-SRH association (Model 1) differs according to both sub-network and demographic group. For the Total sample, there are no Family SN or Friend SN effects, but Group SN is highly significant. The two significant SS effects are both Emotional (Family and Friend), but they are in opposite directions.

When comparing demographic groups, the most striking result is that for three of the four groups, Group SN is positively related to SRH. The absence of a significant Group SN effect for the Unpartnered is in stark contrast to the highly significant effect for the Partnered. Among the Unpartnered, there is only one effect, the marginal benefit of Friend SN for SRH. This marginal effect also occurs among Females. Among Males, Group SN is the only effect, while among the Partnered Family Emotional SS is positively associated with SRH. These positive effects are consistent with the BGBS framework, although there is no indication of causal direction in the positive SN-SRH association.

Several significant negative effects are indicated for Friend Emotional SS and Family Instrumental SS. Together these negative effects suggest that the BGBS 
Table 2:

Study variables by demographic group: means for continuous variables and distributions for categorical variables

\begin{tabular}{|c|c|c|c|c|c|c|c|}
\hline \multirow{2}{*}{$\begin{array}{l}\text { Variable } \\
\text { Sample size }\end{array}$} & \multirow{2}{*}{$\begin{array}{l}\text { Total } \\
1522\end{array}$} & \multicolumn{2}{|l|}{ Males } & \multirow{2}{*}{$\begin{array}{c}\text { Females } \\
857\end{array}$} & \multirow{2}{*}{$\begin{array}{c}\text { Partnered } \\
1141\end{array}$} & \multicolumn{2}{|c|}{ Unpartnered } \\
\hline & & 665 & & & & & 381 \\
\hline SRH (\% Fair/poor) & 14.5 & 14.7 & & 14.2 & 13.5 & & 17.3 \\
\hline Age & 64.5 & 65.6 & $* * *$ & 63.7 & 63.8 & $* * *$ & 66.7 \\
\hline Sex $(\%$ female $)$ & 56.3 & - & & - & 48.1 & $* * *$ & 80.8 \\
\hline Partnership status (\% partnered) & 75.0 & 89.0 & $* * *$ & 64.1 & - & & - \\
\hline Level of education & 3.47 & 3.55 & $*$ & 3.40 & 3.46 & & 3.48 \\
\hline Comfortable with living standard & 1.92 & 1.92 & & 1.92 & 1.86 & $* * *$ & 2.07 \\
\hline Disability limits social activities & 3.22 & 3.16 & & 3.26 & 3.12 & $* * *$ & 3.52 \\
\hline \multicolumn{8}{|l|}{ Family network } \\
\hline Network strength ${ }^{\mathrm{a}}$ & 2.54 & 2.36 & $* * *$ & 2.67 & 2.57 & & 2.44 \\
\hline Family instrumental support ${ }^{\mathrm{a}}$ & 2.42 & 2.29 & $* *$ & 2.53 & 2.40 & & 2.48 \\
\hline Family emotional support ${ }^{\mathrm{a}}$ & 2.18 & 2.06 & $* *$ & 2.27 & 2.14 & $*$ & 2.30 \\
\hline \multicolumn{8}{|l|}{ Friend network } \\
\hline Network strength ${ }^{\mathrm{a}}$ & 2.57 & 2.33 & $* * *$ & 2.76 & 2.48 & $* * *$ & 2.86 \\
\hline Friends instrumental support ${ }^{\mathrm{a}}$ & 2.26 & 2.16 & $* *$ & 2.35 & 2.20 & $* *$ & 2.45 \\
\hline Friends emotional support ${ }^{\mathrm{a}}$ & 2.03 & 1.77 & $* * *$ & 2.23 & 1.95 & $* * *$ & 2.28 \\
\hline \multicolumn{8}{|l|}{ Group network } \\
\hline Time spent in group activities ${ }^{\mathrm{a}}$ & 3.01 & 2.96 & & 3.05 & 2.95 & $*$ & 3.19 \\
\hline
\end{tabular}

Note: Group differences were tested using chi-squared for categorical variables and Student's t for continuous variables. $* p<0.05, * * p<0.01, * * * p<0.001$; ${ }^{\text {a }}$ Refers to previous 4 weeks.

framework may not be entirely appropriate. Rather than stronger SS resulting in better health through positive causation, these negative effects indicate that the dominant causal mechanism is negative reverse causation whereby being in worse health begets stronger SS. These causal mechanisms are confounded in the model.

We explore these opposing mechanisms by dividing the sample into respondents who reported no restrictions on their social activity due to their own health or disability (Unrestricted SN) and those who reported some restrictions (Restricted $\mathrm{SN}$ ). By doing so, we effectively eliminate in the Unrestricted SN model the effects of positive reverse causation of SN and negative reverse causation of Instrumental SS, leaving positive causation and weak negative reverse causation of Emotional SS as possible mechanisms (see Section 3.3). It can be seen in Table 4 that the dominant pattern in the Unrestricted SN sample is indeed positive association, logically attributed to positive causation. This occurs for both Friend SN and Group $\mathrm{SN}$, the sub-networks that can be expected to contribute most to SRH in a socially unrestricted sample; whereas there are no such effects for Family SN.

It can also be seen in Table 4 that the positive effects of Friend SN and Group $\mathrm{SN}$ are again absent among the Unpartnered. For this group, the only effect is a 
Table 3:

Associations with SRH, total sample by demographic group

\begin{tabular}{lccccc}
\hline Variable & Total & Males & Females & Partnered & Un-partnered \\
\hline Age & - & - & - & - & - \\
Sex & & & & & \\
Partnership & & & & & \\
Education & & ++ & & & ++ \\
Comfortable with living standard & +++ & +++ & +++ & +++ & \\
Family SN & & & & & \\
Family Instrumental SS & & & & {$[-]$} & \\
Family Emotional SS & {$[+]$} & & & {$[+]$} & \\
Friend SN & & & {$[+]$} & & \\
Friend Instrumental SS & & & & & \\
Friend Emotional SS & - & & - & - & \\
Group SN & +++ & ++ & ++ & +++ & .075 \\
Sample size & 1522 & 665 & 857 & 1141 & .080 \\
Pseudo R & .070 & .101 & .072 & .070 \\
\hline
\end{tabular}

Note: Based on logistic regression of Fair/poor SRH (Model 1). + positive (beneficial) effect on SRH; - negative (detrimental) effect on SRH; [ ] $p<0.10 ;{ }^{+} p<0.05 ;{ }^{++} p<0.01{ }^{+++} p<0.001$.

Table 4:

Associations with SRH, Unrestricted SN sample ${ }^{a}$ by demographic group

\begin{tabular}{lccccc}
\hline Variable & Total & Males & Females & Partnered & Un-partnered \\
\hline Age & & & & & + \\
Sex & + & & & & \\
Partnership & & & & & \\
Education & +++ & + & ++ & +++ & \\
Comfortable with living standard & ++ & & ++ & + & \\
Family SN & & & & & - \\
Family Instrumental SS & & & & & \\
Family Emotional SS & & & & & \\
Friend SN & + & & ++ & + & \\
Friend Instrumental SS & & & & & \\
Friend Emotional SS & & & & & \\
Group SN & ++ & ++ & & + & \\
Sample size & 800 & 365 & 435 & 615 & \\
Pseudo R & .063 & .058 & .083 & .061 & \\
\hline
\end{tabular}

Note: Based on logistic regression of Good/fair/poor SRH (Model 1). ${ }^{\text {a }}$ Includes respondents whose social activities are never restricted by their health or disability. + positive (beneficial) effect on SRH; - negative (detrimental) effect on SRH. [ ] $p<0.10 ;{ }^{+} p<0.05 ;{ }^{++} p<0.01 ;{ }^{+++} p<0.001$. 
negative effect of Family Emotional SS due to reverse causation: i.e. worse SRH begets greater Emotional SS from family members. The fact that this occurs only among the Unpartnered may be attributable to the absence of a partner which may result in family members serving as confidants in situations of stress or ill health. Thus, the effect of Family Emotional SS differs between the Unpartnered and the Partnered, as the latter can be expected to confide in their partner (the relevant variable was not available).

In the comparison of Males and Females, two highly significant causal effects are contrasted: among Females better SRH stems from having a stronger Friend SN, whereas among Males better SRH stems from having a stronger Group SN. This difference is in line with theories regarding gendered styles of communication, which assert that women are more likely than men to prefer having close and emotionally satisfying relationships (Cross and Madson 1997).

The models for the Restricted SN sample are shown in Table 5. For this sample, all three causal mechanisms are applicable. Model 1 shows a positive SN-SRH association for Group SN for Females and the Partnered, as well as for the Total (the model for the Unpartnered is non-significant). It is not possible to distinguish between positive causation and positive reverse causation. For these groups, there is also evidence of a negative SN-SRH association in relation to Friend Emotional SS, which suggests that ill health may be the subject of confidential disclosure (negative reverse causation).

The applicability of the causal mechanisms is likely to be related to the degree of SN restriction. Thus for the Restricted SN sample, further analysis was undertaken to take the frequency of SN restriction into account (Model 2), thereby essentially accounting for reverse causation in the positive association. It is expected that due to the inclusion of SN restriction, the positive SN-SRH association will be weakened.

Table 5 (Model 2) shows that this is indeed the case. The frequency of SN restriction is very highly significant for all demographic groups, which indicates that reverse causation contributes substantially to the positive SN-SRH association across the sample. The previously strongly positive Group SN-SRH association is reduced to a weak association found only among the Partnered. In other words, the positive association between Group SN and SRH is accounted for by the degree of SN restriction due to the ego's health or disability, or his or her ability to physically access group activities. Comparing Model 1 with Model 2, only one effect persists: the negative association of Friend Emotional SS with SRH for Females. The addition of interaction terms (not shown) did not change these results appreciably. Thus, it would appear that once reverse causation has been accounted for in the BGBS framework, only the model for Females shows a significant SNSRH association in the form of negative reverse causation for Friend Emotional SS. Females in worse health confide in more friends. 
Table 5:

Associations with SRH, Restricted SN sample ${ }^{\text {a }}$ by demographic group

\begin{tabular}{|c|c|c|c|c|c|c|c|c|c|c|}
\hline \multirow[b]{2}{*}{ Variable } & \multicolumn{5}{|c|}{ Model 1} & \multicolumn{5}{|c|}{ Model 2} \\
\hline & $\mathbf{T}$ & $\mathbf{M}$ & $\mathbf{F}$ & $\mathbf{P}$ & $\mathbf{U}$ & $\mathbf{T}$ & $\mathbf{M}$ & $\mathbf{F}$ & $\mathbf{P}$ & $\mathbf{U}$ \\
\hline $\begin{array}{l}\text { Age } \\
\text { Sex } \\
\text { Partnership } \\
\text { Education }\end{array}$ & - & ++ & - & - & & - & {$[+]$} & {$[-]$} & - & \\
\hline $\begin{array}{l}\text { Education } \\
\text { Comfortable with living standard } \\
\text { SN restriction }\end{array}$ & +++ & $\begin{array}{l}++ \\
++\end{array}$ & ++ & +++ & & $\begin{array}{l}++ \\
-\end{array}$ & $\begin{array}{l}{[+]} \\
{[+]} \\
-\end{array}$ & $\stackrel{[+]}{-}$ & $\begin{array}{l}++ \\
-\end{array}$ & - \\
\hline $\begin{array}{l}\text { Family SN } \\
\text { Family Instrumental SS } \\
\text { Family Emotional SS }\end{array}$ & & & & {$[+]$} & & & & & & \\
\hline $\begin{array}{l}\text { Friend SN } \\
\text { Friend Instrumental SS } \\
\text { Friend Emotional SS }\end{array}$ & - & & - & - & & & & - & {$[-]$} & \\
\hline Group SN & ++ & & + & ++ & & & & & {$[+]$} & \\
\hline $\begin{array}{l}\text { Sample size } \\
\text { Pseudo } \mathrm{R}^{2}\end{array}$ & $\begin{array}{l}722 \\
.050\end{array}$ & $\begin{array}{l}300 \\
.074\end{array}$ & $\begin{array}{l}422 \\
.055\end{array}$ & $\begin{array}{l}526 \\
.073\end{array}$ & & $\begin{array}{l}722 \\
.208\end{array}$ & $\begin{array}{l}300 \\
.240\end{array}$ & $\begin{array}{l}422 \\
.201\end{array}$ & $\begin{array}{l}526 \\
.242\end{array}$ & $\begin{array}{l}196 \\
.188\end{array}$ \\
\hline
\end{tabular}

Note: Based on logistic regression of Fair/poor SRH. ${ }^{\text {a }}$ Includes respondents whose social activities are to some extent restricted by their health or disability. Model 1 for Unpartnered (U) is non-significant. + positive (beneficial) effect on SRH; - negative (detrimental) effect on SRH. [ ] $p<0.10 ;{ }^{+} p<0.05 ;^{++} p<0.01 ;^{+++} p<0.001$.

\section{Discussion}

This study sought to identify differences in the SN-SRH association among complementary population groups and exclusive social sub-networks, with both delineated by demographic characteristics. Apparently plausible findings were obtained, including a highly significant positive Group SN-SRH association and a negative association between Friend Emotional SS and SRH. These findings may be directly compared with many of those in the literature, and are in some cases consistent.

However, the analyses also involved the consideration of three causal mechanisms underlying the observed associations: positive causation, positive reverse causation, and negative reverse causation. After identifying as far as possible the competing mechanisms in the total sample, the sample was divided into two sub-samples according to the frequency with which the respondent's social activity was restricted by his or her own health or disability. This enabled some causal effects to be estimated, changing the interpretation of the initial findings. 


\subsection{Unrestricted and Restricted social networks}

The findings for the Unrestricted SN and Restricted SN samples differ in important respects. In the Unrestricted SN sample, for which there is logically no positive reverse causation (health does not restrict social activity) and no negative reverse causation between either SN or Instrumental SS and SRH (healthy people do not need disability-related activity or support), positive effects were identified, indicating a causal SN-SRH association: i.e. stronger social networks beget better SRH. These effects were confined to the Friend SN and Group SN among the Total, Males, Females, and the Partnered. Among the Unpartnered, a negative causal effect was found for Family Emotional SS, indicating that unpartnered people in worse health confide in more family members (see Section 6.3). For this healthy subsample, there were no effects of Family SN or of Friend Instrumental or Emotional SS. The inapplicability of Instrumental SS is confirmed in the model estimates.

In the Restricted SN sample, a different story emerges. Model 1 shows that both positive and negative associations exist. Again, Group SN is positively associated with SRH among several groups (Total, Females, and Partnered), but for the same three groups Friend Emotional SS is negatively associated with SRH, indicating that people in worse health confide in more friends. However, when the frequency of SN restriction is included (Model 2), accounting for positive reverse causation, the positive Group SN-SRH association is no longer significant, except weakly so among the Partnered; while the negative Friend Emotional SS-SRH association remains only for Females. In other words, the two initial effects, which also feature in the total sample, are largely explained in the Restricted SN sample by the degree of $\mathrm{SN}$ restriction. Very few significant effects remain.

The finding of negative reverse causation in the Friend Emotional SS-SRH association among Females (i.e. that those in worse health confide in more friends) is consistent with socio-emotional selectivity theory (Carstensen 1995), which predicts that a greater sense of limited remaining life expectancy (assumed here to stem from worse SRH) results in greater selectivity in favour of emotionally rewarding relationships; or, in other words, a negative association with Emotional SS. There is no evidence that this selectivity reduces network size to the extent that a positive effect of Friend SN on SRH occurs. In this model, this is to be expected because the inclusion of SN restriction essentially takes account of any positive network size effect (due to reverse causation) arising from this corollary of the theory. An indication of a weak negative effect of Friend SN among the Partnered (negative reverse causation) suggests that more friends visit partnered people in worse health more frequently.

The findings for the two samples have implications for the applicability of the BGBS framework for the study of the SN-SRH association. The final models for the Unrestricted SN sample indicate that for the 'healthy', if not for the Unpartnered, the BGBS framework of positive causation is applicable. However, the models for the Restricted SN sample show that there is no positive causal effect of SN on SRH, except marginally among the Partnered. For this 'unhealthy' sample, the BGBS 
framework does not apply. This is problematic because research on the SN-SRH association is more concerned more the unhealthy than the healthy, or at least with the comparison of these groups. The validity of much of the published literature on the SN-SRH association based on cross-sectional data may also be called into question. It is likely that the findings of such studies are a weighted average of the effects of the three causal mechanisms demonstrated here; evidence for this can be seen in comparing Tables 3-5.

\subsection{Males and Females}

The models for Males and Females differ in both the Unrestricted SN and the Restricted SN samples. In the Unrestricted SN sample, the positive SN-SRH association derives from the Group SN for Males and from the Friend SN for Females. In the Restricted SN sample, Friend Emotional SS is significant for Females but not for Males. These sex differences can be attributed to gender. Research on gendered relationship preferences has suggested that men generally do not seek to extend their network of friends (often not beyond their spouse), whereas women maintain larger and more multifaceted networks (Antonucci and Akiyama 1987b). It has also been previously reported that men derive greater health benefits from group activities than women (Caetano et al. 2013). This may be because the non-group social networks of men are more likely than those of women to be classified as 'low exchange' or 'restricted' (calculated from Fiori and Jager 2012 Table 1). Gendered socialisation leads to differences in the meaning of relationships, with men's relationships tending to be more practical, instrumental, and informational; and female relationships being more relational and emotional (Olson and Shultz 1994; Cross and Madson 1997).

When the degree of SN restriction is taken into account (Model 2 for the Restricted SN sample), no effects are found among Males, but the negative association between Friend Emotional SS and SRH persists among Females. The finding that this effect occurs only among Females can be attributed to the preference among women for emotionally satisfying relationships (Cross and Madson 1997). Stemming logically from negative reverse causation, this effect indicates that whatever their level of $\mathrm{SN}$ restriction due to their own health or disability, Females reporting worse SRH confide in more friends.

These findings suggest that friendship, rather than the family, plays an important role in women's health, and that women place considerable value on emotional support from friends. The results also indicate that men can benefit from less emotional relationships (through group activities), but that men in ill health gain little or no benefit from social networks or social support, which would suggest that men have a certain degree of social vulnerability. 


\subsection{Partnered and Unpartnered}

Substantial differences also exist between the Partnered and the Unpartnered. In the Unrestricted SN sample, Family Emotional SS is negatively associated with SRH among the Unpartnered, and is this group's only effect, indicating that those with worse SRH confide in more family members. Among the Partnered in the Unrestricted SN sample, both Friend SN and Group SN show positive effects: i.e. having stronger social networks begets better SRH. In the Restricted SN sample, Model 2 shows that there are no effects among the Unpartnered and only weak effects among the Partnered, with the latter finding indicating that Group SN maintains some level of positive causal (beneficial) effect on SRH, while having worse SRH results in a stronger Friend SN based on disability-related visits.

These findings can be attributed in part to structural differences in the social networks of partnered and unpartnered older people. Different life course experiences play a fundamental role. The social networks of partnered people tend to be family-oriented; the partner and children are the main sources of support. Furthermore, the partner, where present, is not only the main source of instrumental support (especially in disability), but for males in particular is often the only source of emotional support (Antonucci and Akiyama 1987b). Our data do not capture the role of the partner (or of other co-resident family members). This may explain why we find no effects for social support among the Partnered.

The social reliance of partnered people on their partner and family may help to explain why Friend SN and Group SN are positively associated with SRH among the Partnered in the Unrestricted SN sample. Group networks in particular, but also friend networks, generally differ from the family networks of partnered people, offering the opportunity for different types of social relationships with a greater diversity of people. Moreover, participation in group networks is voluntary, involves fewer responsibilities and obligations, takes place outside the home, and may involve exercise and outdoor activities; factors which are associated with better SRH or physical health (Berkman et al. 2000; Litwin 2006; Cherry et al. 2013). Partnered people who rely less on their partner and family network, and maintain stronger friend and group networks can be expected to benefit in terms of better health.

In the Restricted sample, after taking account of the degree of SN restriction, the positive effect of Group SN on SRH is weakly maintained. However, the association between Friend SN and SRH is weakly negative, which (as already noted) indicates that more friends visit partnered people in worse health more frequently. That these effects occur only among the Partnered may be an artefact of sample size, but it is also likely that some partnered people in ill health benefit from the presence of the partner as a facilitator of both participation in group activities and visits by friends.

Previous research has found that for people without partners, networks tend to be relatively friend-oriented, and that friends can play an important role as sources of support (Seeman and Berkman 1988; Cornwell et al. 2008). Our finding for the total sample (Table 3) that Friend SN is weakly positively associated with SRH 
among the Unpartnered is consistent with these existing findings. However, in the Unrestricted SN sample, having worse SRH (of a form that does not restrict social activity) leads to the Unpartnered confiding in more family members, rather than in friends. This finding may be due to the fact that $81 \%$ of the Unpartnered in this sub-sample have previously been partnered, which suggests that most have children in whom they may confide. The small sample size precluded meaningful analysis of the Unpartnered group in Restricted SN sample.

These findings suggest that for the healthy and partnered, one key to better SRH is the strength of friend and group social networks. Maintaining these networks while in ill health is also beneficial. In the case of the friend social network, the net effect is negative, i.e. having worse SRH begets a stronger Friend SN. However, having friends is a prerequisite for having visits from friends. There are no positive effects for the Unpartnered, which may suggest a degree of social vulnerability.

\subsection{Family, Friend, and Group networks}

Among the three networks, positive causal effects on SRH are found for the Friend and Group networks, but not for the Family network (which is itself noteworthy). In the Unrestricted SN sample, having a stronger Friend SN and Group SN both contribute to better health, while in the Restricted SN sample the contribution of Group SN is also present. The voluntary nature of friendship (Litwin 2006; Huxhold et al. 2013) and group activities may help to explain these findings; Litwin (2006) also found a beneficial effect for frequency of contact with friends.

The positive effects of Group SN and Friend SN are consistent with the Cherry et al. (2013) finding that time spent outside the home in social activities (including through club/organisation membership) was more important than social support in predicting self-reported physical health (though, perhaps more informatively, this study also found club membership to be 'predictive' of objective health). We find that the Group SN and Friend SN effects are almost entirely confined to the Unrestricted SN sample. In the Restricted SN sample, Model 2 shows only a marginal effect of Group SN among the Partnered, indicating that the positive association of Group SN with SRH (Model 1) is due to reverse causation. Thus, the positive Group SN-SRH association would appear to be explained almost entirely by the degree of SN restriction due to ego's health or disability, in other words, by the ability to physically access group activities.

Thus the positive effect of Group SN, which features so strongly in the models for the total sample (Table 3), is almost entirely confined to the Unrestricted SN sample; in other words, to the healthy. If the benefits of group activities, demonstrated by the positive causal relationship found in the healthy, are to be extended to the unhealthy, better physical access is required. This interpretation is supported by the indication that access may be facilitated by the presence of a partner (Section 6.3). 


\subsection{The role of $\mathrm{SN}$ restriction in the models}

This study has underlined the importance of reverse causation in understanding the $\mathrm{SN}-\mathrm{SRH}$ association at older ages. Through the use of the variable measuring SN restriction, we have been able to identify three causal mechanisms (Table 1): positive causation, positive reverse causation, and negative reverse causation.

There are, of course, more blurred distinctions between causation and reverse causation than those represented in these models. Here it is important to recall that SRH is a much broader and more multifaceted construct than the restriction of social activity due to health or disability, and that SRH is imperfectly related to objective health (Idler et al. 1999); moreover, unmeasured variables are also likely to contribute to blurred distinctions. Though positive reverse causation has been eliminated or taken into account in terms of SN restriction in the models, a degree of reverse causation stemming from the broader construct is likely to exert an influence. Positive causation and positive reverse causation are reinforcing: to this extent the models are likely to overestimate the role of positive causation in the $\mathrm{SN}-\mathrm{SRH}$ association.

Furthermore, positive reverse causation and negative reverse causation are counterbalancing: the estimated effects of SRH on SN/SS are net effects. Whenever negative reverse causation is present, the strength of the SN-SRH association is underestimated. Thus, any statistical evidence of negative reverse causation is likely to be substantially underestimated, as it must significantly outweigh both positive causation and positive reverse causation. Friend Emotional SS among Females is a case in point.

Longitudinal data would be needed to accurately quantify these separate effects. This study has highlighted the existence of the contribution of positive reverse causation and negative reverse causation to the SN-SRH association in a sample for which this is possible.

\subsection{Limitations, strengths, and future research}

A main limitation of the study is the sample on which it is based. The study population, members of National Seniors Australia, is unlikely to be entirely representative of the wider Australian population. Thus, though we have taken account of known sources of potential bias (age and education), the extent to which the results can be generalised is limited. This limitation is, however, of reduced importance in this study, because we compare only the significance patterns among models for demographic groups.

Limitations in the comparability of models for the Partnered and the Unpartnered arise from the lack of availability of variables concerning the partner's role in the social network and social support of partnered respondents. This is likely the reason why the Unpartnered exhibit unique patterns of significance. Future research might 
usefully consider the inclusion of the partner and other co-resident family members in the family network.

The major strength of the study is our ability to address causation. This was possible through a direct measure of the degree to which own health or disability restricts social activity. By dividing the sample on this continuum, thereby logically eliminating competing mechanisms and by taking the degree of SN restriction into account, it was possible to identify causal and reverse causal effects. To our knowledge, the analysis is unique.

Finally, the findings for the Unrestricted SN and the Restricted SN samples differ considerably. Notably, positive causal effects of SN on SRH are confined to the 'healthy' Unrestricted SN sample, while no such effects are found for the 'unhealthy' Restricted SN sample (with one weakly significant exception). This calls into question the validity of analyses that do not take positive and negative reverse causation into account in the SN-SRH association, and may explain some of the confusion among the extant findings. Furthermore, differences between complementary demographic groups have been shown to be masked in the Total sample. This suggests that analyses that treat demographic variables purely as covariates routinely ignore important differences deriving from the demographic social-structural conditions. Differences between sub-networks are also found to be substantial in this study, which suggests that the widespread tendency to conflate family and friends is not appropriate. These differences underline the importance of demography in defining the context and the boundaries of the SN-SRH association, and in understanding that association. The findings of this study have considerable relevance for future research.

\section{Acknowledgements}

This research was supported by an Australian Research Council (ARC) Linkage Grant (project number LP09909674) in collaboration with National Seniors Australia, and by the ARC Centre of Excellence in Population Ageing Research (project number CE110001029); and was approved by the Australian National University Human Research Ethics Committee (Protocol 2009/360). The findings and views reported in this paper are those of the author(s) and not those of the supporting agencies. We thank the participants in our research. We also thank two anonymous reviewers and the editors for their useful comments.

\section{References}

Adams, K. B., Leibbrandt, S. and Moon, H. 2011. A critical review of the literature on social and leisure activity and wellbeing in later life. Ageing and Society 31(04): 683-712. doi:10.1017/S0144686X10001091. 
Ajrouch, K. J., Blandon, A. Y. and Antonucci, T. C. 2005. Social Networks among men and women: the effects of age and socioeconomic status. The Journals of Gerontology Series B: Psychological Sciences and Social Sciences 60(6): S311-S317. doi:10.1093/geronb/60.6.S311.

Antonucci, T. C. and Akiyama, H. 1987a. Social Networks in adult life and a preliminary examination of the convoy model. Journal of Gerontology 42(5): 519-527. doi:10.1093/geronj/42.5.519.

Antonucci, T. and Akiyama, H. 1987b. An examination of sex differences in social support among older men and women. Sex Roles 17(11-12): 737-749. doi:10.1007/bf00287685.

Antonucci, T., Akiyama, H. and Lansford, J. E. 1998. Negative Effects of close social relations. Family Relations 47(4): 379-383.

Ashida, S. and Heaney, C. A. 2008. Differential associations of social support and social connectedness with structural features of social networks and the health status of older adults. Journal of Aging and Health 20(7): 872-893. doi:10.1177/0898264308324626.

Australian Bureau of Statistics 2011. Census of population and housing, Australia: Canberra.

Barnes, J. 1954. Class and committees in a Norwegian Island Parish. Human Relations 7: 39-58.

Benyamini, Y., Leventhal, E. A. and Leventhal, H. 2003. Elderly people's ratings of the importance of health-related factors to their self-assessments of health. Social Science and Medicine 56(8): 1661-68.

Berkman, L. F., Glass, T., Brissette, I. and Seeman, T. E. 2000. From social integration to health: Durkheim in the new millennium. Social Science and Medicine 51: 843-857.

Booth, H., Crawford, H., Windsor, T. D., Rioseco, P., Bradley, C., Chen, L. and Ackland, R. 2013. Staying connected: Social engagement and wellbeing among mature age Australians. Canberra: National Seniors Productive Ageing Centre.

Bowling, A. and Grundy, E. 1998. The association between social networks and mortality in later life. Reviews in Clinical Gerontology 8(4): 353-361.

Burke, K. E., Schnittger, R., O’Dea, B., Buckley, V., Wharton, J. P. and Lawlor, B. A. 2012. Factors associated with perceived health in older adult Irish population. Aging and Mental Health 16(3): 288-295.

Caetano, S. C., Silva, C. and Vettore, M. V. 2013. Gender differences in the association of perceived social support and social network with self-rated health status among older adults: a population-based study in Brazil. BMC Geriatrics 13(122): 1-14.

Carstensen, L. L. 1995. Evidence for a life-span theory of socioemotional selectivity. Current Directions in Psychological Science 4(5): 151-156. doi:10.2307/20182356.

Cheng, S.-T. and Chan, A. C. M. 2006. Social support and self-rated health revisited: Is there a gender difference in later life? Social Science and Medicine 63(1): 118-122. doi:10.1016/j.socscimed.2005.12.004.

Cherry, K. E., Walker, E. J., Brown, J. S., Volaufova, J., LaMotte, L. R., Welsh, D. A., Su, L. J., Jazwinski, S. M., Ellis, R., Wood, R. H. and Frisard, M. I. Adults in the Louisiana healthy aging study social engagement and health in younger, older, and oldest-old. Journal of Applied Gerontology 32(1): 57-75. doi:10.1177/0733464811409034.

Cornwell, B., Laumann, E. O. and Schumm, L. P. 2008. The social connectedness of older adults: a national profile. American Sociological Review 73(2): 185-203. 
Cross, S. E. and Madson, L. 1997. Models of the self: self-construals and gender. Psychological Bulletin 122(1): 5-37.

Duncan, T. and Frankenberg, E. 2000. The measurement and interpretation of health in social surveys. RAND Working Paper Series, Santa Monica: CA.

Dupertuis, L. L., Aldwin, C. M. and Bossé, R. 2001. Does the source of support matter for different health outcomes? findings from the normative aging study. Journal of Aging and Health 13(4): 494-510.

Ferlander, S. and Mäkinen, I. H. 2009. Social capital, gender and self-rated health. Evidence from the Moscow Health Survey 2004. Social Science and Medicine 69(9): 1323-1332. doi:10.1016/j.socscimed.2009.08.009.

Finch, J. and Mason, J. 1993 Negotiating family responsibilities. London: Routledge.

Fiori, K. L. and Jager, J. 2012. The impact of social support networks on mental and physical health in the transition to older adulthood: A longitudinal, patterncentered approach. International Journal of Behavioral Development 36(2): 117-129. doi: $10.1177 / 0165025411424089$.

García, E. L., Banegas, J. R., Pérez-Regadera, A. G., Cabrera, R. H. and Rodríguez-Artalejo, F. 2005. Social network and health-related quality of life in older adults: a population-based study in Spain. Quality of Life Research 14(2): 511-520. doi:10.1007/s11136-004-5329-z.

Gilmour, H. 2012. Social participation and the health and well-being of Canadian seniors. Health Reports 23(4): 23-32.

Gjonca, A., Tomassini, C., Toson, B. and Smallwood, S. 2005. Sex differences in mortality, a comparison of the United Kingdom and other developed countries. Health Statistics Quarterly 26: 6-16.

Grundy, E. and Sloggett, A. 2003. Health inequalities in the older population: the role of personal capital, social resources and socio-economic circumstances. Social Science and Medicine 56(5): 935-947.

Huxhold, O., Miche, M. and Schüz, B. 2013. Benefits of having friends in older ages: differential effects of informal social activities on well-being in middle-aged and older adults. Journals of Gerontology Series B: Psychological Sciences and Social Sciences 69(3): 366-375. doi:10.1093/geronb/gbt029.

Idler, E. L. and Benyamini, Y. 1997. Self-rated health and mortality: a review of twenty-seven community studies. Journal of Health and Social Behavior 38(1): 21.

Idler, E. L., Hudson, S. V. and Howard Leventhal 1999. The meanings of self-ratings of health: a qualitative and quantitative approach. Research on Aging 21(3): 458-476. doi: $10.1177 / 0164027599213006$.

Jylhä, M. 2009. What is self-rated health and why does it predict mortality? Towards a unified conceptual model. Social Science and Medicine 69(3): 307-316. doi:10.1016/j.socscimed.2009.05.013.

Kadushin, C. 2012 Understanding Social Networks: Theories, Concepts and Findings. New York: Oxford University Press.

Kalmijn, M. and Vermunt, J. K. 2007. Homogeneity of social networks by age and marital status: a multilevel analysis of ego-centered networks. Social Networks 29: 25-43.

Kavanagh, A. M., Bentley, R., Turrell, G., Broom, D. H. and Subramanian, S. V. 2006. Does gender modify associations between self-rated health and the social and economic 
characteristics of local environments? Journal of Epidemiology and Community Health 60(6): 490-495. doi: 10.1136/jech.2005.043562.

Kennedy, B. P., Kawachi, I., Glass, R. and Prothrow-Stith, D. 1998. Income distribution, socioeconomic status, and self-rated health in the United States: multilevel analysis. British Medical Journal 317: 917-921.

Lennartsson, C. 1999. Social ties and health among the very old in Sweden. Research on Aging 21(5): 657-681.

Lindenberger, U., Singer, T. and Baltes, P. B. 2002. Longitudinal selectivity in aging populations: Separating mortality-associated versus experimental components in the Berlin Aging Study (BASE). Journal of Gerontology: Psychological Sciences 57B: P474P482. doi:10.1093/geronb/57.6.P474.

Litwin, H. 2006. Social networks and self-rated health: a cross-cultural examination among older Israelis. Journal of Aging and Health 18(3): 335-358. doi:10.1177/0898264305280982.

Litwin, H. and Shiovitz-Ezra, S. 2011. Social Network type and subjective wellbeing in a national sample of older Americans. The Gerontologist 51(3): 379-388. doi:10.1093/geront/gnq094.

Lundberg, O. and Manderbacka, K. 1996. Assessing reliability of a measure of self-rated health. Scandinavian Journal of Public Health 24(3): 218-224. doi:10.1177/140349489602400314.

Manderbacka, K. 2013. Examining what self-rated health question is understood to mean by respondents. Scandinavian Journal of Public Health 26(2): 145-153.

Mirowsky, J. and Ross, C. E. 2008. Education and self-rated health, cumulative advantage and its rising importance. Research on Aging 30(1): 93-122. doi:10.1177/0164027507309649.

McLaughlin, D., Vagenas, D., Pachana, N. A., Begum, N. and Dobson, A. 2010. Gender differences in social network size and satisfaction in adults in their 70s. Journal of Health Psychology 15(5): 671-679. doi:10.1177/1359105310368177.

McPherson, M., Smith-Lovin, L. and Cook, J. M. 2001. Birds of a feather: Homophily in social networks. Annual Review of Sociology 27: 415-444.

Melchior, M., Berkman, L. F., Niedhammer, I., Chea, M. and Goldberg, M. 2003. Social relations and self-reported health: a prospective analysis of the French Gazel cohort. Social Science and Medicine 56(8): 1817-1830.

Norstrand, J. A., Glicksman, A., Lubben, J. and Kleban, M. 2012. The role of the social environment on physical and mental health of older adults. Journal of Housing For the Elderly 26(1-3): 290-307.

Nummela, O., Sulander, T., Rahkonen, O., Karisto, A. and Uutela, A. 2008. Social participation, trust and self-rated health: a study among ageing people in urban, semi-urban and rural settings. Health and Place 14(2): 243-253. doi:10.1016/j.healthplace.2007.06.006.

Okamoto, K. and Tanaka, Y. 2004. Gender differences in the relationship between social support and subjective health among elderly persons in Japan. Preventive Medicine 38(3): $318-322$. 
Olson, D. A. and Shultz, K. S. 1994. Gender differences in the dimensionality of social support. Journal of Applied Social Psychology 24(14): 1221-1232. doi:10.1111/j.15591816.1994.tb00555.x/.

Pollack, C. E. and von dem Knesebeck, O. 2004. Social capital and health among the aged: comparisons between the United States and Germany. Health and Place 10(4): 383-391. doi:10.1016/j.healthplace.2004.08.008.

Salmon, C. T. and Nichols, J. S. 1983. The Next-Birthday Method of respondent selection. Public Opinion Quarterly 47: 270-276.

Sargent-Cox, K. A., Anstey, K. J. and Luszcz, M. A. 2008. Determinants of self-rated health items with different points of reference: implications for health measurement of older adults. Journal of Aging and Health 20(6): 739-761.

Scott, J. 2000. Social Network Analysis: A Handbook. London: SAGE publications.

Seeman, T. and Berkman, L. F. 1988. Structural characteristics of social networks and their relationship with social support in the elderly: who provides support. Social Science and Medicine 26(7) 737-749.

Shye, D., Mullooly, J. P., Freeborn, D. K. and Pope, C. R. 1995. Gender differences in the relationship between social network support and mortality: a longitudinal study of an elderly cohort. Social Science and Medicine 41(7): 935-947. doi:10.1016/02779536(94)00404-H.

Smith, K. P. and Christakis, N. A. 2008. Social Networks and health. Annual Review of Sociology 34(1): 405-418.

Wenger, G. C., Scott, A. and Patterson, N. 2000. How important is parenthood? Childlessness and support in old age in England. Ageing and Society 20(2): 161-182.

White, A. M., Philogene, G. S., Fine, L. and Sinha, S. 2009. Social support and self-reported health status of older adults in the United States. American Journal of Public Health 99(10): 1872-1878.

Wolf, D. A. 1994. The elderly and their kin: patterns of availability and access. In Demography of Aging, ed. S. H. Preston and L. G. Martin, pp. 146-194. Washington, DC: National Academy Press.

Zunzunegui, M. V., Koné, A., Johri, M., Béland, F., Wolfson, C. and Bergman, H. 2004. Social Networks and self-rated health in two French-speaking Canadian community dwelling populations over 65. Social Science and Medicine 58(10): 2069-2081. 
